Despite the tentative nature of most of the findings, it is fairly safe to say that group incentive payment schemes are likely to yield the best results when: (a) groups are small, compact and stable; $(b)$ the members of a group are well matched and engaged on the same type of work; (c) the form of payment is simple and the workers are able to translate output into earnings as work proceeds throughout the week ; (d) there are no anomalies in rates and standards within a group or between groups; (e) output depends on the worker rather than on the machine; $(f)$ the operational cycle is fairly short and repetitive ; $(g)$ variations in the quality and flow of material are reduced to a minimum ; $(h)$ the group is able to earn a reasonable bonus. (It is generally recognized that the bonus should be about one-third of the time rate, but the optimum rate has never been determined); (i) there is an atmosphere of mutual trust and understanding between management and workers.

When the operations in a department are too varied and irregular for sectional payment the incentive scheme may have to be related to departmental output. This is fairly straightforward when the product is uniform, but it is not easy when there are several products and the proportions vary from week to week. Under such conditions it is necessary to determine the number of man-hours per unit of output for each type of product and convert them to a common basis. The same procedure can, of course, be applied to factory output, though the scheme will lose some of its incentive value as its remoteness from individual effort increases. On the other hand, a payment scheme based on the total output of a department or factory is less costly in administrative and clerical work than one based on the output of individuals or small groups. It also reduces or eliminates many sources of friction and facilitates the movement or interchange of workers.

\section{SCIENCE AND THE LONDON COUNTY COUNCIL}

$F^{\mathrm{E}}$ W London citizens are aware of the considerable If amount of work done on their behalf by the Scientific Branch of the Public Health Department of the London County Council. Yet this branch, under the scientific adviser, Dr. S. G. Burgess, has a staff of fifty-nine which includes twenty-six graduates. The function of the branch is to advise all departments of the Council on scientific matters. The work of the branch is carried out in three groups of laboratories, the headquarters laboratories at the County Hall and those at the Northern and Southern Outfall Works situated at Beckton and Crossness. A recent report* of the scientific adviser describes the work carried out in 1955 . During the year, 30,335 samples were taken in connexion with renewed investigations into sewage treatments, paints and surface coatings, building materials, school meals, drinking water, swimming baths, ventilation of vehicular tunnels, lubricating oils, polishes, disinfectants, insecticides and fungicides and other topics.

New work was commenced on air pollution, regular observations being taken at twenty sites. The

* Iondon County Council: Public Health Department, Scientiflc Branch. Annual Report of the Scientific Adviser for the year 1955. observations made showed a marked difference between rural and urban conditions, and also variation in different localities within the County due to local sources of pollution. Seasonal fluctuations were clearly marked at all sites, the generally higher level of pollution in winter being due to the combined effect of increased coal consumption and the occurrence of meteorological conditions unfavourable to the dispersion of chimney gases. When the results are examined over a period of years they show a slight reduction in the amounts of tarry matter and smoke; this can be accounted for by the replacement of many domestic fires by the more efficient combustion units of blocks of flats. A distinction could be drawn between the effects of domestic and industrial combustion. For example, on week-days the smoke to sulphur ratio is at a minimum during the night, when most domestic fires die down, while some industry keeps running. At week-ends, when a greater proportion of industry is closed down, and domestic fires are burning for longer periods, this ratio is found to be higher than for comparable periods during the week.

A new series of investigations has been made into the effects of discharging trade effluents into sewers. 940 samples were taken, and of these 193 , or 20.4 per cent, were considered to contravene the appropriate Act. Of this number, 86 were discharges with varying degrees of acidity. The acids identified included hydrochloric from pickling shops, nitric acid from photo-engravers, sulphuric acid from plating and anodizing shops, acetic acid from pickle manufacturers, citric acid from soft drink manufacturers and formic acid used for descaling and cleaning cookers. Also found were mixed organic acids similar to acetic acid from distilleries, bakeries and other works using fermentation processes. Wastes containing suspended matter in sufficient quantity to cause deposition and partial blockage of the sewers were those containing fats, thick mineral oils, tar, paint residues, vegetable fibres and tannery wastes.

In two cases, complete blockage of pipe sewers had taken place. One of these was caused by the wet grinding of stone and the other by a mixture of siliceous material and caustic metasilicate from a vitreous enamelling works. Of wastes containing inflammable solvents, the larger number were from garages, consisting of mixtures of waste lubricating oil with varying quantities of paraffin and petroleum spirit. One sample contained 37 per cent by volume of petroleum spirit. Three wastes from paint works contained white spirit or solvent naphtha. Another waste consisting of wash and cooling water, from a biological products plant, contained traces of monochlorbenzene and was sufficiently hot and of such total volume that the solvent, vaporized in the sewer, rendered the atmosphere unbreathable. Waste heat contained in process and cooling waters was another source of nuisance and possible dangers in the sewers. About forty cases of discharges above the $110^{\circ} \mathrm{F}$. temperature limit laid down in the Act were investigated during the year. The adviser recommends that manufacturers should consider ways and means of recovering such waste heat in the interests of efficiency and economy.

In many cases following the examination of samples which contravene the Act, officers of the Scientific Branch and of the Chief Engineer's Department visit. the premises to bring to the notice of the occupiers the particular nature of the offence and to discuss the problems involved in treating the discharges. 